\title{
On the Renormalization Group Techniques for the Cubic-Quintic Duffing Equation
}

\author{
Supratim Das
Department of Applied Mathematics, University of Calcutta, 92 Acharya Prafulla Chandra Road, Kolkata-700009, India. \\ supratimiitkgp@gmail.com
}

Keywords: Duffing oscillator; Renormalization group; indicial term; Multiple time scales.

\begin{abstract}
We apply the renormalization group techniques for solving the nonlinear cubic-quintic Duffing equation in the presence of an external periodic, non-autonomous force with an additional damping term. We also make a comparative study with the multiple-time scale approach and show that the correction to the frequency is the same.
\end{abstract}

\section{Introduction}

In recent times the method of renormalization group (RG) has been employed [1]-[4] through the introduction of a set of modified variables to arrive at the elimination of secular terms. The theory of RG has rich connections with quantum field theory and is considered to be a very powerful tool to handle the so-called 'divergences' of quantum electrodynamics [5]. It has several applications in the areas of phase transitions and critical phenomena $[6,7]$ and asymptotic analysis of a variety of perturbed ordinary and partial differential equations $[1,2,8]$. RG argument has also been used to study jump phenomena and stability in nonlinear oscillators [3].

In this article we discuss the RG method for the cubic-quintic Duffing oscillator, proposed by Chua [9], in the presence of an external periodic (non-autonomous) force with an additional damping term moving in a sextic potential

$$
\ddot{x}+\alpha \dot{x}+\omega_{0}^{2} x+\nu x^{3}+\sigma x^{5}=\Omega \cos \omega t .
$$

In [9] perturbative analytical techniques were proposed to derive approximate periodic solutions and period-amplitude relations. Duffing oscillator with odd nonlinearities has been studied in the literature to model the nonlinear dynamics of various systems including that of a slender elastica, the compound $\mathrm{KdV}$, the propagation of a short electromagnetic pulse in a nonlinear medium (see for instance, [9]-[14] and references therein) and position or momentum-dependent mass schemes [15, 16]. In particular, Linstedt-Poincaré techniques were applied for the specific case of quintic Duffing equation by Ramos [11] by an artificial parameter method. The extended scheme (1) describes a classical particle in a triple-well potential for appropriate choices of parameters. In the phase portrait at most five equilibrium points exist for it revealing a wide variety of interesting dynamical behaviour.

A modified variable $\tau$ is defined by [17]

$$
\tau=\bar{\omega} t+\theta \Rightarrow x(t) \rightarrow z(\tau) \equiv z(\bar{\omega} t+\theta) .
$$

In the following we set $\nu=\lambda \epsilon, \sigma=\eta \epsilon$ and $\Omega=f \epsilon$ where an intention is to carry out a perturbation analysis in terms of the infinitesimal quantity $\epsilon<<1$. We thus express equation (1) in the form

$$
\bar{\omega}^{2} z^{\prime \prime}+\alpha \bar{\omega} z^{\prime}+\omega_{0}^{2} z+\lambda \epsilon z^{3}+\eta \epsilon z^{5}=f \epsilon \cos \left(\frac{\omega}{\bar{\omega}}(\tau-\theta)\right), \quad z^{\prime}=\frac{d z}{d \tau}
$$

We look for an expansion of both $z$ and $\bar{\omega}$. To first order in $\epsilon$ we can write

$$
\begin{aligned}
z(\tau) & =z_{0}(\tau)+\epsilon z_{1}(\tau)+O\left(\epsilon^{2}\right), \\
\bar{\omega} & =\bar{\omega}_{0}+\epsilon \bar{\omega}_{1}+O\left(\epsilon^{2}\right) .
\end{aligned}
$$


Taking $\bar{\omega}_{0}=\omega_{0}$ so that $\bar{\omega}=\omega_{0}+\epsilon \bar{\omega}_{1}+O\left(\epsilon^{2}\right)$, we substitute (4) into equation (3) and then collecting the terms of like powers in the perturbation parameter $\epsilon$ (up to order $\epsilon$ ) yields the flow of equations

$$
\begin{aligned}
& \epsilon^{0} \quad: \quad z_{0}^{\prime \prime}+\frac{\alpha}{\omega_{0}} z_{0}^{\prime}+z_{0}=0, \\
& \epsilon^{1}: \quad z_{1}^{\prime \prime}+\frac{\alpha}{\omega_{0}} z_{1}^{\prime}+z_{1}=-\frac{2 \bar{\omega}_{1}}{\omega_{0}} z_{0}^{\prime}-\frac{\alpha \bar{\omega}_{1}}{\omega_{0}^{2}} z_{0}^{\prime}-\frac{\lambda}{\omega_{0}^{2}} z_{0}^{3}-\frac{\eta}{\omega_{0}^{2}} z_{0}^{5}+\frac{f}{\omega_{0}^{2}} \cos \left(\frac{\omega}{\bar{\omega}}(\tau-\theta)\right) .
\end{aligned}
$$

A natural assumption is that the coefficient $\alpha$ of the damping term is non-negative and hence we discuss the following two cases.

Case-I : $\alpha=0$

In the absence of damping term, we set $\alpha=0$ and in this case the solution of equation (5) reads

$$
z_{0}=a \cos D \tau
$$

where $a$ is a constant and using this solution equation (6) can be reduced to

$$
\begin{aligned}
z_{1}^{\prime \prime}+z_{1}= & \frac{a}{8 \omega_{0}^{2}}\left(16 \omega_{0} \bar{\omega}_{1}-6 \lambda a^{2}-5 \eta a^{4}\right) \cos \tau-\frac{a^{3}}{16 \omega_{0}^{2}}\left(4 \lambda+5 \eta a^{2}\right) \cos 3 \tau \\
& -\frac{\eta a^{5}}{16 \omega_{0}^{2}} \cos 5 \tau+\frac{f}{\omega_{0}^{2}} \cos \left(\frac{\omega}{\bar{\omega}}(\tau-\theta)\right) .
\end{aligned}
$$

The solution of equation (8) would be a cosine function as equation (7) if the right hand side of (8) were zero. The particular solution of (8) can be obtained as

$$
\begin{aligned}
z= & \frac{a}{32 \omega_{0}^{2}}\left(16 \omega_{0} \bar{\omega}_{1}-6 \lambda a^{2}-5 \eta a^{4}\right) \cos \tau+\frac{a^{3}}{128 \omega_{0}^{2}}(4 \lambda \\
& \left.+5 \eta a^{2}\right) \cos 3 \tau+\frac{\eta a^{5}}{384 \omega_{0}^{2}} \cos 5 \tau+\frac{f}{\omega_{0}^{2}-\omega^{2}} \cos \left(\frac{\omega}{\bar{\omega}}(\tau-\theta)\right) \\
& +\frac{a}{16 \omega_{0}^{2}}\left(16 \omega_{0} \bar{\omega}_{1}-6 \lambda a^{2}-5 \eta a^{4}\right) \tau \sin \tau
\end{aligned}
$$

implying that $z(\tau)$ is given by

$$
\begin{aligned}
z=a \cos \tau+\epsilon[ & \frac{a}{32 \omega_{0}^{2}}\left(16 \omega_{0} \bar{\omega}_{1}-6 \lambda a^{2}-5 \eta a^{4}\right) \cos \tau+\frac{a^{3}}{128 \omega_{0}^{2}}(4 \lambda \\
& \left.+5 \eta a^{2}\right) \cos 3 \tau+\frac{\eta a^{5}}{384 \omega_{0}^{2}} \cos 5 \tau+\frac{f}{\omega_{0}^{2}-\omega^{2}} \cos \left(\frac{\omega}{\bar{\omega}}(\tau-\theta)\right) \\
& \left.+\frac{a}{16 \omega_{0}^{2}}\left(16 \omega_{0} \bar{\omega}_{1}-6 \lambda a^{2}-5 \eta a^{4}\right) \tau \sin \tau\right]
\end{aligned}
$$

where the last term is the secular or the growth term. For $\bar{\omega}_{1}=\frac{6 \lambda a^{2}+5 \eta a^{4}}{16 \omega_{0}}$ the secular term vanishes. In the Lindstedt approach, elimination of the secular terms is done in each step of the power series by recursively fixing $\bar{\omega}_{1}, \bar{\omega}_{2}$ and so on. However, as is well known, there are some difficulties with the convergence of the Lindstedt expansion although such a disadvantage is not always serious for a physical problem [18]. In the following we adopt instead the RG approach that introduces an arbitrary time scale $\mu$ and the RG constants are adjusted to eliminate terms like $\tau-\mu, \tau^{2}-\mu^{2}$ so that we dealt with a finite form for $z$.

Renormalization group (RG) analysis Let us keep $\bar{\omega}_{1} \neq \frac{6 \lambda a^{2}+5 \eta a^{4}}{16 \omega_{0}}$ and apply the RG technique $[1,3]$ on (10) to get bounded solution of (3). Introducing an arbitrary time scale $\mu$ and express $\tau$ as $\tau=[\tau-\mu]+[\mu-0]$ with the intention that the unwanted divergences are reduced only historical 
curiosities $(\mu-0)$ and we are left concerned only with the present time scale $(t-\mu)$ i.e. a singularityfree time. Towards this end we introduce renormalization parameters $Z_{1}(\mu)$ and $Z_{2}(\mu)$ in a perturbative manner such that

$$
\begin{aligned}
& a(0)=a_{0}=Z_{1}(\mu) a(\mu)=\left(1+\bar{A}_{1} \epsilon\right) a(\mu), \\
& \theta(0)=\theta(\mu)+Z_{2}=\theta(\mu)+\bar{B}_{1} \epsilon .
\end{aligned}
$$

We utilize $\bar{A}_{1}, \bar{B}_{1}$ in such a way that the secular terms are made to vanish.

Denoting

$$
\bar{a} \equiv a(\mu), \quad \bar{\theta}=\theta(\mu)
$$

we get from (10) up to order $\epsilon$

$$
\begin{aligned}
z= & \left(1+\bar{A}_{1} \epsilon\right) \bar{a} \cos \left(\bar{\omega} t+\bar{\theta}+\bar{B}_{1} \epsilon\right)+\epsilon\left[\frac{\bar{a}}{32 \omega_{0}^{2}}\left(16 \omega_{0} \bar{\omega}_{1}-6 \lambda \bar{a}^{2}-5 \eta \bar{a}^{4}\right) \cos (\bar{\omega} t+\bar{\theta})\right. \\
& +\frac{\bar{a}^{3}}{128 \omega_{0}^{2}}\left(4 \lambda+5 \eta \bar{a}^{2}\right) \cos 3(\bar{\omega} t+\bar{\theta})+\frac{\eta \bar{a}^{5}}{384 \omega_{0}^{2}} \cos 5(\bar{\omega} t+\bar{\theta})+\frac{f}{\omega_{0}^{2}-\omega^{2}} \cos \omega t \\
& \left.+\frac{\bar{a}}{16 \omega_{0}^{2}}\left(16 \omega_{0} \bar{\omega}_{1}-6 \lambda \bar{a}^{2}-5 \eta \bar{a}^{4}\right) \tau \sin (\bar{\omega} t+\bar{\theta})\right] \\
= & \bar{a} \cos (\bar{\omega} t+\bar{\theta})+\epsilon\left[\bar{A}_{1} \bar{a} \cos (\bar{\omega} t+\bar{\theta})-\bar{B}_{1} \bar{a} \sin (\bar{\omega} t+\bar{\theta})+\frac{\bar{a}}{32 \omega_{0}^{2}}\left(16 \omega_{0} \bar{\omega}_{1}\right.\right. \\
& \left.-6 \lambda \bar{a}^{2}-5 \eta \bar{a}^{4}\right) \cos (\bar{\omega} t+\bar{\theta})+\frac{\bar{a}^{3}}{128 \omega_{0}^{2}}\left(4 \lambda+5 \eta \bar{a}^{2}\right) \cos 3(\bar{\omega} t+\bar{\theta}) \\
& +\frac{\eta \bar{a}^{5}}{384 \omega_{0}^{2}} \cos 5(\bar{\omega} t+\bar{\theta})+\frac{f}{\omega_{0}^{2}-\omega^{2}} \cos \omega t \\
& \left.+\frac{\bar{a}}{16 \omega_{0}^{2}}\left(16 \omega_{0} \bar{\omega}_{1}-6 \lambda \bar{a}^{2}-5 \eta \bar{a}^{4}\right)\{(\tau-\mu)+\mu\} \sin (\bar{\omega} t+\bar{\theta})\right] .
\end{aligned}
$$

Inspection reveals that the divergent term vanish for the conditions

$$
\bar{A}_{1}=0, \quad \bar{B}_{1}=\frac{\mu}{16 \omega_{0}^{2}}\left(16 \omega_{0} \bar{\omega}_{1}-6 \lambda \bar{a}^{2}-5 \eta \bar{a}^{4}\right) .
$$

For this choices of $\bar{A}_{1}$ and $\bar{B}_{1}$ solution $z$ becomes

$$
\begin{aligned}
z= & \bar{a} \cos (\bar{\omega} t+\bar{\theta})+\epsilon\left[\frac{\bar{a}}{32 \omega_{0}^{2}}\left(16 \omega_{0} \bar{\omega}_{1}-6 \lambda \bar{a}^{2}-5 \eta \bar{a}^{4}\right) \cos (\bar{\omega} t+\bar{\theta})\right. \\
& +\frac{\bar{a}^{3}}{128 \omega_{0}^{2}}\left(4 \lambda+5 \eta \bar{a}^{2}\right) \cos 3(\bar{\omega} t+\bar{\theta})+\frac{\eta \bar{a}^{5}}{384 \omega_{0}^{2}} \cos 5(\bar{\omega} t+\bar{\theta}) \\
& \left.+\frac{f}{\omega_{0}^{2}-\omega^{2}} \cos \omega t+\frac{\bar{a}}{16 \omega_{0}^{2}}\left(16 \omega_{0} \bar{\omega}_{1}-6 \lambda \bar{a}^{2}-5 \eta \bar{a}^{4}\right)(\tau-\mu) \sin (\bar{\omega} t+\bar{\theta})\right] .
\end{aligned}
$$

Since the dynamics needs to be independent of the renormalization scale i.e. $\frac{\partial z}{\partial \mu}=0$ which implies

$$
\frac{d \bar{\theta}}{d \mu}=-\frac{\epsilon}{16 \omega_{0}^{2}}\left(16 \omega_{0} \bar{\omega}_{1}-6 \lambda \bar{a}^{2}-5 \eta \bar{a}^{4}\right), \frac{d \bar{a}}{d \mu}=0
$$

and this gives

$$
\bar{\theta}=-\frac{\epsilon \mu}{16 \omega_{0}^{2}}\left(16 \omega_{0} \bar{\omega}_{1}-6 \lambda \bar{a}^{2}-5 \eta \bar{a}^{4}\right), \bar{a}=\text { constant } .
$$


Substituting $\mu=\tau$ in (15) yielding the renormaliztion expansion of $z$ up to order $\epsilon$

$$
\begin{aligned}
z= & a \cos \left\{\bar{\omega}\left(1-\frac{\epsilon}{16 \omega_{0}^{2}}\left(16 \omega_{0} \bar{\omega}_{1}-6 \lambda a^{2}-5 \eta a^{4}\right)\right) t\right\}+\epsilon\left[\frac { a } { 3 2 \omega _ { 0 } ^ { 2 } } \left(16 \omega_{0} \bar{\omega}_{1}\right.\right. \\
& \left.-6 \lambda a^{2}-5 \eta a^{4}\right) \cos (\bar{\omega} t+\theta)+\frac{a^{3}}{128 \omega_{0}^{2}}\left(4 \lambda+5 \eta a^{2}\right) \cos 3(\bar{\omega} t+\theta) \\
& \left.+\frac{\eta a^{5}}{384 \omega_{0}^{2}} \cos 5(\bar{\omega} t+\theta)+\frac{f}{\omega_{0}^{2}-\omega^{2}} \cos \omega t\right]
\end{aligned}
$$

Clearly (18) is free from any divergent term.

Multiple time scales Consider two separate independent time scales which are $\tau_{0}=\epsilon^{0} \tau=\tau$ and $\tau_{1}=\epsilon^{1} \tau_{0}=\epsilon \tau$. We express $z=z\left(\tau_{0}, \tau_{1}\right)$ and employ the expansion

$$
z\left(\tau_{0}, \tau_{1}\right)=\sum_{n=0}^{\infty} \epsilon^{n} z_{n}\left(\tau_{0}, \tau_{1}\right)
$$

Thus for $\alpha=0$ we obtain from equation (3) the result

$$
\begin{aligned}
& \left(\omega_{0}^{2}+2 \epsilon \omega_{0} \bar{\omega}_{1}\right)\left(\frac{\partial^{2} z_{0}}{\partial \tau_{0}^{2}}+\epsilon \frac{\partial^{2} z_{1}}{\partial \tau_{0}^{2}}+2 \epsilon \frac{\partial^{2} z_{0}}{\partial \tau_{0} \partial \tau_{1}}\right) \\
& +\omega_{0}^{2}\left(z_{0}+\epsilon z_{1}\right)+\lambda \epsilon z_{0}^{3}+\eta \epsilon z_{0}^{5}=f \epsilon \cos \left(\frac{\omega}{\bar{\omega}}\left(\tau_{0}-\theta\right)\right) .
\end{aligned}
$$

To zeroth-order in $\epsilon$ we have $\frac{\partial^{2} z_{0}}{\partial \tau_{0}^{2}}+z_{0}=0$ whose solution is given by

$$
z_{0}=a \cos \tau_{0} ; \quad \tau_{0}=\bar{\omega}, t_{0}+\theta
$$

where $a$ and $\theta$ can both be functions of $\left(\tau_{0}, \tau_{1}\right)$.

On the other hand for the first-order in $\epsilon$, we isolate from (20)

$$
\frac{\partial^{2} z_{1}}{\partial \tau_{0}^{2}}+z_{1}+2 \frac{\partial^{2} z_{0}}{\partial \tau_{0} \partial \tau_{1}}=-\frac{2 \bar{\omega}_{1}}{\omega_{0}} \frac{\partial^{2} z_{0}}{\partial \tau_{0}^{2}}-\frac{\lambda}{\omega_{0}^{2}} z_{0}^{3}-\frac{\eta}{\omega_{0}^{2}} z_{0}^{5}+\frac{f}{\omega_{0}^{2}} \cos \left(\frac{\omega}{\bar{\omega}}\left(\tau_{0}-\theta\right)\right) .
$$

By observing that $\frac{\partial^{2} z_{0}}{\partial \tau_{0}^{2}}=-a \cos \tau_{0}$ and $\frac{\partial^{2} z_{0}}{\partial \tau_{0} \partial \tau_{1}}=-a \cos (\bar{\omega} t+\theta) \frac{\partial \theta}{\partial \tau_{1}}-\sin (\bar{\omega} t+\theta) \frac{\partial a}{\partial \tau_{1}}$, equation (22) gives

$$
\begin{aligned}
\frac{\partial^{2} z_{1}}{\partial \tau_{0}^{2}}+z_{1}= & \frac{f}{\omega_{0}^{2}} \cos \left(\frac{\omega}{\bar{\omega}}\left(\tau_{0}-\theta\right)\right)+2 a \cos (\bar{\omega} t+\theta) \frac{\partial \theta}{\partial \tau_{1}}+\frac{2 \bar{\omega}_{1}}{\omega_{0}} a \cos (\bar{\omega} t+\theta) \\
& -\frac{\lambda a^{3}}{4 \omega_{0}^{2}}[\cos 3(\bar{\omega} t+\theta)+3 \cos (\bar{\omega} t+\theta)]-\frac{\eta a^{5}}{\omega_{0}^{2}}\left[\frac{1}{16} \cos 5(\bar{\omega} t+\theta)\right. \\
& \left.+\frac{5}{16} \cos 3(\bar{\omega} t+\theta)+\frac{5}{8} \cos (\bar{\omega} t+\theta)\right]+2 \frac{\partial a}{\partial \tau_{1}} \sin (\bar{\omega} t+\theta) .
\end{aligned}
$$

Equating now to zero the coefficients of the sine, cosine terms of right hand side gives

$$
\frac{\partial a}{\partial \tau_{1}}=0, \quad \frac{\partial \theta}{\partial \tau_{1}}=-\frac{16 \omega_{0} \bar{\omega}_{1}-6 \lambda a^{2}-5 \eta a^{4}}{16 \omega_{0}^{2}}
$$

while the remaining terms imply

$$
\frac{\partial^{2} z_{1}}{\partial \tau_{0}^{2}}+z_{1}=\frac{f}{\omega_{0}^{2}} \cos \left(\frac{\omega}{\bar{\omega}}\left(\tau_{0}-\theta\right)\right)-\left(\frac{\lambda a^{3}}{4 \omega_{0}^{2}}+\frac{5 \eta a^{5}}{16 \omega_{0}^{2}}\right) \cos 3(\bar{\omega} t+\theta)-\frac{\eta a^{5}}{\omega_{0}^{2}} \cos 5(\bar{\omega} t+\theta) .
$$


From (24) and using $\tau_{1}=\epsilon\left(\bar{\omega} t_{0}+\theta\right)$ we obtain up to order $\epsilon$,

$$
a=\text { constant }, \quad \theta=-\frac{16 \omega_{0} \bar{\omega}_{1}-6 \lambda a^{2}-5 \eta a^{4}}{16 \omega_{0}^{2}}\left(\bar{\omega} t_{0}+\theta\right) \epsilon .
$$

The final expression for $z$ according to equation (19), takes the form

$$
z=a \cos \left[\bar{\omega}\left(1-\frac{16 \omega_{0} \bar{\omega}_{1}-6 \lambda a^{2}-5 \eta a^{4}}{16 \omega_{0}^{2}}\right) t_{0}\right]+\epsilon z_{1}\left(t_{0}, t_{1}\right)+\ldots
$$

Note that the correction to the frequency of this solution is same as the solution (18).

\section{Case-II : $\alpha>0$}

In this case we solve equation (3) in the presence of damping term i.e. for $\alpha>0$. The solution of equation $(5)$ reads

$$
z_{0}=a e^{-\frac{\alpha}{2 \omega_{0}} \tau} \cos D \tau,
$$

where $a$ is a constant and $D$ is given by

$$
D=\sqrt{1-\frac{\alpha^{2}}{4 \omega_{0}^{2}}}
$$

When the solution (28) is used, equation (6) takes the form

$$
\begin{aligned}
z_{1}^{\prime \prime}+\frac{\alpha}{\omega_{0}} z_{1}^{\prime}+z_{1}= & \frac{\left(4 \omega_{0}^{2}-\alpha^{2}\right) \bar{\omega}_{1} a}{2 \omega_{0}^{3}} e^{-\frac{\alpha}{2 \omega_{0}} \tau} \cos D \tau-\frac{D \alpha \bar{\omega}_{1} a}{\omega_{0}^{2}} e^{-\frac{\alpha}{2 \omega_{0}} \tau} \sin D \tau \\
& -\frac{\lambda a^{3}}{4 \omega_{0}^{2}} e^{-\frac{3 \alpha}{2 \omega_{0}} \tau}(3 \cos D \tau+\cos 3 D \tau) \\
& -\frac{\eta a^{5}}{8 \omega_{0}^{2}} e^{-\frac{5 \alpha}{2 \omega_{0}} \tau}\left(5 \cos D \tau+\frac{5}{2} \cos 3 D \tau+\frac{1}{2} \cos 5 D \tau\right) \\
& +\frac{f}{\omega_{0}^{2}} \cos \left(\frac{\omega}{\bar{\omega}}(\tau-\theta)\right)
\end{aligned}
$$

Particular integral of equation (30) can be determined as

$$
\begin{aligned}
z_{1}= & \frac{\left(4 \omega_{0}^{2}-\alpha^{2}\right) \bar{\omega}_{1} a}{4 \omega_{0}^{3} D} e^{-\frac{\alpha}{2 \omega_{0}} \tau} \tau \sin D \tau+\frac{\alpha \bar{\omega}_{1} a}{2 \omega_{0}^{2}} e^{-\frac{\alpha}{2 \omega_{0}} \tau} \tau \cos D \tau \\
& -\frac{3 \lambda a^{3}}{16 \omega_{0}^{2} \alpha} e^{-\frac{3 \alpha}{2 \omega_{0}} \tau}\left(\alpha \cos D \tau-2 \omega_{0} \sin D \tau\right) \\
& -\frac{\lambda a^{3}}{16 \omega_{0}^{2}\left(16 \omega_{0}^{2}-3 \alpha^{2}\right)} e^{-\frac{3 \alpha}{2 \omega_{0}} \tau}\left\{\left(-8 \omega_{0}^{2}+3 \alpha^{2}\right) \cos 3 D \tau-6 \alpha \omega_{0} D \sin 3 D \tau\right\} \\
& -\frac{5 \eta a^{5}}{8 \alpha\left(3 \alpha^{2}+4 \omega_{0}^{2}\right)} e^{-\frac{5 \alpha}{2 \omega_{0}} \tau}\left(\alpha \cos D \tau-\omega_{0} D \sin D \tau\right) \\
& -\frac{5 \eta a^{5}}{128 \omega_{0}^{2}\left(4 \omega_{0}^{2}+3 \alpha^{2}\right)} e^{-\frac{5 \alpha}{2 \omega_{0}} \tau}\left\{\left(-4 \omega_{0}^{2}+3 \alpha^{2}\right) \cos 3 D \tau-6 \alpha \omega_{0} D \sin 3 D \tau\right\} \\
& -\frac{5 \eta a^{5}}{128 \omega_{0}^{2}\left(36 \omega_{0}^{2}-5 \alpha^{2}\right)} e^{-\frac{5 \alpha}{2 \omega_{0}} \tau}\left\{\left(-12 \omega_{0}^{2}+5 \alpha^{2}\right) \cos 5 D \tau-2 \alpha \omega_{0} D \sin 5 D \tau\right\} \\
& +\frac{f \bar{\omega}^{2}}{\omega_{0}^{2}\left(\bar{\omega}^{2}+\omega^{2}\right)^{2}+\alpha^{2} \omega^{2} \bar{\omega}^{2}}\left\{\left(\bar{\omega}^{2}+\omega^{2}\right) \cos \left(\frac{\omega}{\bar{\omega}}(\tau-\theta)\right)+\frac{\alpha \omega \bar{\omega}}{\omega_{0}} \sin \left(\frac{\omega}{\bar{\omega}}(\tau-\theta)\right)\right\}
\end{aligned}
$$


which indicates that the solution $z(\tau)$ of equation (3) up to order $\epsilon$ can be put as

$$
\begin{aligned}
z= & a e^{-\frac{\alpha}{2 \omega_{0}} \tau} \cos D \tau+\epsilon\left[\frac{\left(4 \omega_{0}^{2}-\alpha^{2}\right) \bar{\omega}_{1} a}{4 \omega_{0}^{3} D} e^{-\frac{\alpha}{2 \omega_{0}} \tau} \tau \sin D \tau+\frac{\alpha \bar{\omega}_{1} a}{2 \omega_{0}^{2}} e^{-\frac{\alpha}{2 \omega_{0}} \tau} \tau \cos D \tau\right. \\
& -\frac{3 \lambda a^{3}}{16 \omega_{0}^{2} \alpha} e^{-\frac{3 \alpha}{2 \omega_{0}} \tau}\left(\alpha \cos D \tau-2 \omega_{0} \sin D \tau\right) \\
& -\frac{\lambda a^{3}}{16 \omega_{0}^{2}\left(16 \omega_{0}^{2}-3 \alpha^{2}\right)} e^{-\frac{3 \alpha}{2 \omega_{0}} \tau}\left\{\left(-8 \omega_{0}^{2}+3 \alpha^{2}\right) \cos 3 D \tau-6 \alpha \omega_{0} D \sin 3 D \tau\right\} \\
& -\frac{5 \eta a^{5}}{8 \alpha\left(3 \alpha^{2}+4 \omega_{0}^{2}\right)} e^{-\frac{5 \alpha}{2 \omega_{0}} \tau}\left(\alpha \cos D \tau-\omega_{0} D \sin D \tau\right) \\
& -\frac{5 \eta a^{5}}{128 \omega_{0}^{2}\left(4 \omega_{0}^{2}+3 \alpha^{2}\right)} e^{-\frac{5 \alpha}{2 \omega_{0}} \tau}\left\{\left(-4 \omega_{0}^{2}+3 \alpha^{2}\right) \cos 3 D \tau-6 \alpha \omega_{0} D \sin 3 D \tau\right\} \\
& -\frac{5 \eta a^{5}}{128 \omega_{0}^{2}\left(36 \omega_{0}^{2}-5 \alpha^{2}\right)} e^{-\frac{5 \alpha}{2 \omega_{0}} \tau}\left\{\left(-12 \omega_{0}^{2}+5 \alpha^{2}\right) \cos 5 D \tau-2 \alpha \omega_{0} D \sin 5 D \tau\right\} \\
& \left.+\frac{f \bar{\omega}^{2}}{\omega_{0}^{2}\left(\bar{\omega}^{2}+\omega^{2}\right)^{2}+\alpha^{2} \omega^{2} \bar{\omega}^{2}}\left\{\left(\bar{\omega}^{2}+\omega^{2}\right) \cos \left(\frac{\omega}{\bar{\omega}}(\tau-\theta)\right)+\frac{\alpha \omega \bar{\omega}}{\omega_{0}} \sin \left(\frac{\omega}{\bar{\omega}}(\tau-\theta)\right)\right\}\right] .
\end{aligned}
$$

Clearly this solution is free from any divergent term.

\section{Summary}

In this work we have employed the RG approach to investigate the dynamical behaviour of a cubicquintic Duffing oscillator endowed with an external periodic non-autonomous force. The RG approach ensures a divergence free result. A comparative study with the multiple-time scale approach shows that the correction to the frequency is the same. We also obtained a perturbative solution of the same equation with an additional damping term.

\section{Acknowledgements}

I thank Prof. Bijan Kumar Bagchi for guidance. I also thank CSIR, India for providing a Senior Research Fellowship.

\section{References}

[1] L.Y. Chen, N. Goldenfeld, Y. Oono, Renormalization group and singular perturbations: Multiple scales, boundary layers, and reductive perturbation theory, Phys Rev. 54 (1996) 376-394.

[2] L.Y. Chen, N. Goldenfeld, Y. Oono, Renormalization group theory for global asymptotic analysis, Phys. Rev. Lett. 73(10) (1994) 1311.

[3] D. Banerjee, J.K. Bhattacharjee, Analyzing jump phenomena and stability in nonlinear oscillators using renormalization group arguments, Am. J. Phys. 78(2) (2010) 142-149.

[4] R.E.L. DeVille et al., Analysis of a renormalization group method and normal form theory for perturbed ordinary differential equations, Physica D: Nonlinear Phenomena. 237(8) (2008) 10291052 .

[5] N. Goldenfeld, D. Pines, Westview Press, Lectures on phase transitions and the Renormalization Group, 1992. 
[6] D.J. Amit, V.M. Mayor, Field Theory; the Renormalization Group and Critical Phenomena:Graphs to Computers, World Scientific Press, 2005.

[7] J. Zinn-Justin, Quantum Field Theory and Critical Phenomena, Oxford, Clarendon, 1989.

[8] T. Tao et al., Renormalization Group Method for Soliton Evolution in a Perturbed KdV Equation, Chin. Phys. Lett. 26(6) (2009) 060501.

[9] V. Chua, Cubic-Quintic Duffing Oscillators. (unpublished)

[10] S.K. Lai et al., Applied Mathematical Modelling, Simulation and Computation for Engineering and Environmental systems. 33 (2009) 852.

[11] J.I. Ramos, On Linstedt-Poincaré techniques for the quintic Duffing equation, Applied Mathematics and Computation. 193(2) (2007) 303-310.

[12] C.W. Lim et al., Nonlinear free vibration of an elastically-restrained beam with a point mass via the Newton-harmonic balancing approach, International Journal of Nonlinear Sciences and Numerical simulation. 10(5) (2009) 661-674.

[13] A. Beléndez et al., Analytical approximate solutions for the cubic-quintic Duffing oscillator in terms of elementary functions, Journal of Applied Mathematics. 2012 (2012) 286290.

[14] M. Lakshmanan, S. Rajasekar, Nonlinear dynamics : Integrability, Chaos and Patterns, Advanced Texts in Physics, Springer-Verlag, Berlin, 2003.

[15] B. Bagchi et al., Nonlinear dynamics of a position-dependent mass-driven Duffing-type oscillator, J. Phys. A: Math. Theor. 46(3) (2012) 032001.

[16] V. Chithika Ruby, M. Senthilvelan, M. Lakshmanan, Exact quantization of a PT-symmetric (reversible) Liénard-type nonlinear oscillator, J. Phys. A: Math. Theor. 45(38) (2012) 382002.

[17] A. Lindstedt, Abh. K. Akad. Wiss. St. Petersburg. 31(4) (1882).

[18] N. Minorsky, Nonlinear oscillations, Melbourne : Krieger, 1974. 